\title{
Radiative decays of dynamically generated charmed baryons
}

\author{
D. Gamermann, ${ }^{*}$ C. E. Jiménez-Tejero, and A. Ramos \\ Departament d'Estructura i Constituents de la Matèria and Institut de Ciències del Cosmos, \\ Universitat de Barcelona, Avda. Diagonal 647, E-08028 Barcelona, Spain
}

(Received 25 November 2010; published 21 April 2011)

\begin{abstract}
In this work we study the radiative decay of dynamically generated $J^{P}=\frac{1^{-}}{2}$ charm baryons into the ground state $J^{P}=\frac{1^{+}}{2}$ baryons. Since different theoretical interpretations of these baryonic resonances and, in particular, of the $\Lambda_{c}(2595)$, give different predictions, a precise experimental measurement of these decays would be an important step for understanding their nature.
\end{abstract}

DOI: 10.1103/PhysRevD.83.074018

PACS numbers: 13.30.Ce

\section{INTRODUCTION}

The research on charm hadron physics has experienced a renewed interest in recent years, after the discovery of a few meson states which could not be easily described as $q \bar{q}$ states of quark model potentials, such as the $D_{s 0}(2317)$, the $D_{s 1}(2460)$ [1-4], in the open-charm sector, and the $X(3872)$ [5-8], the $X(3940)[9,10]$, the $Y(3940)$ [11,12], or the $Z(3930)$ [13], in the hidden-charm sector. There has also been a substantial amount of theoretical progress leading to the observation that some of these states could be interpreted alternatively as molecules [14-25], tetraquarks [26-29], or hybrids [30]. A similar situation is found in the baryon sector. Some of the newly observed charmed baryons states [31-39], such as the $J^{P}=$ $\frac{1^{-}}{2} \Lambda_{c}$ (2595) or the $J^{P}=\frac{3^{-}}{2} \Lambda_{c}(2625)$, can be interpreted as meson-baryon molecules that can be generated dynamically within coupled-channel unitary schemes [40-48].

The decay modes of a resonance may provide a way of learning about the nature of the state: whether it fits in the conventional $q q q$-baryon or $q \bar{q}$-meson picture or it has a more exotic interpretation. In particular, the radiative decays into lower lying states may represent a significant part of the decay width when the hadronic modes are suppressed by phase-space restrictions or/and small values of the coupling constants. Note that electromagnetic transitions are also useful in the determination of the quantum numbers of states decaying into a final hadron with well-established quantum numbers.

Radiative decays of ground state heavy flavored baryons have been studied within many different approaches, like heavy hadron chiral perturbation theory (HHCPT) [49-52], an approach which combines heavy quark (HQ) symmetry with the chiral symmetry in the light sector, employing light-cone QCD sum rules [53], supplementing heavy quark symmetry with a $\mathrm{SU}\left(2 N_{f}\right) \times \mathrm{O}(3)$ symmetry in the light diquark system [54], or implementing also dynamical effects for the internal quark structure of the hadrons within the relativistic three-quark model (RTQM) $[55,56]$ or other nonrelativistic quark models [57-59].

*daniel.gamermann@ific.uv.es
Many of the former approaches have also been applied to obtain the radiative decays of excited heavy flavored baryons, such as the $\Lambda_{c}(2595)$. A first qualitative estimation was given in Ref. [60], where the HHCPT formalism was extended to include the lowest lying excited baryon doublet, $\Lambda_{c}$ (2595) $\left(J^{P}=\frac{1^{-}}{2}\right)$ and $\Lambda_{c}(2625)\left(J^{P}=\frac{3^{-}}{2}\right)$. The results of Ref. [54] were obtained by exploiting the alternative light diquark $\mathrm{SU}\left(2 N_{f}\right) \times \mathrm{O}(3)$ symmetry. Radiative decays of excited charmed baryons were also calculated within the relativistic quark model of Refs. [55,56], as well as using light-cone QCD sum rules in Ref. [61]. All these works consider the excited heavy baryon as being an orbital excitation of the three-quark $Q q q$ system with a unit of angular momentum inserted between the heavy quark $Q$ and the light diquark $q q$. A different perspective is provided by the model of Ref. [62], for example. This model considers the excited $\Lambda_{c}(2595)$ and $\Lambda_{c}(2625)$ as being $D^{*} N$ bound systems in the first excited state of a harmonic potential adjusted phenomenologically to reproduce the spin-averaged excitation energy. Another example is the model of Ref. [63] which considers the radiative decay of the $\Lambda_{c}(2940)$ in a hadronic $D^{*} N$ molecular picture.

In the present work we study the radiative decays of excited baryons in the $C=1$ sector. We focus on the resonances $\Lambda_{c}(2595), \Sigma_{c}(2800), \Xi_{c}(2790)$, and $\Xi_{c}(2970)$, all having $J^{P}=\frac{1^{-}}{2}$, which have been generated as mesonbaryon molecular states on several models based on coupled-channel dynamics [40-47]. The radiative transitions to baryons of the $J^{P}=\frac{1^{+}}{2}$ ground state multiplet proceeds, as we will see, through the coupling of the photon to the various meson-baryon components of the resonance, as determined by the coupled-channel dynamical model of Ref. [47]. This is essentially different from the quark models for which, in the heavy quark limit, the photon only couples to the light diquark system. Therefore, a precise measurement of the radiative decays of excited heavy flavored baryons would help in understanding their internal structure.

The radiative decays of dynamically generated charm mesons have already been addressed recently. In [64-66], the radiative decay of the $D_{s 0}^{*}(2317)$ meson has been 
studied from the point of view that it is generated dynamically mainly from the interaction of the $D$ meson with a kaon. Also radiative decays of the puzzling $X(3872)$ have been calculated from the point of view that this state is a $D^{*} \bar{D}+$ c.c. molecule [67]. More recently, many different radiative decays of the controversial $X, Y$, and $Z$ states have been analyzed assuming that their structure is determined by the interactions of two vector mesons [68-70].

This work is organized as follows: in the next section we explain the model for generating dynamically the $J^{P}=\frac{1^{-}}{2}$ baryon resonances from the interaction of ground state baryons with pseudoscalar mesons. In Sec. III we present the framework for evaluating the diagrams needed in the calculation of the radiative decays of the resonances. The results of the calculation are presented and discussed in Sec. IV and in Sec. V we present a brief overview and our conclusions.

\section{THE DYNAMICALLY GENERATED STATES}

In this section we will review the coupled-channel approach employed in [47] to obtain dynamically generated open-charm baryon resonances.

The scattering amplitudes $T$, which describe the scattering of the pseudoscalar meson fields off the ground state baryon fields can be obtained by solving the well-known Lipmann-Schwinger equation, which schematically reads

$$
T=V+V J T .
$$

The loop function $J$ is the product of the meson and baryon single-particle propagators, and the scattering kernel $V$ describes the interaction between the pseudoscalar mesons and the ground state baryons. Following the original work of Hofmann and Lutz [43], we identify a $t$-channel exchange of vector mesons as the driving force for the $s$-wave scattering between pseudoscalar mesons in 16-plet and baryons in 20-plet representations. The scattering kernel takes the form (see [43] for details)

$$
\begin{aligned}
& V_{i j}^{(I, S, C)}\left(p_{i}, q_{i}, p_{j}, q_{j}\right) \\
& =\frac{g^{2}}{4} \sum_{V \in[16]} C_{i j ; V}^{(I, S, C)} \bar{u}\left(p_{j}\right) \gamma^{\mu}\left(g_{\mu \nu}-\frac{\left(q_{i}-q_{j}\right)_{\mu}\left(q_{i}-q_{j}\right)_{\nu}}{m_{V}^{2}}\right) \\
& \quad \times \frac{1}{t-m_{V}^{2}}\left(q_{i}+q_{j}\right)^{\nu} u\left(p_{i}\right),
\end{aligned}
$$

where the sum runs over all vector mesons of the SU(4) 16-plet, $\left(\rho, K^{*}, \bar{K}^{*}, \omega, \phi, D^{*}, D_{s}^{*}, \bar{D}^{*}, \bar{D}_{s}^{*}, J / \Psi\right), m_{V}$ is the mass of the exchanged vector meson, $g$ is the universal vector meson coupling constant, $p_{i}, q_{i}\left(p_{j}, q_{j}\right)$ are the fourmomenta of the incoming (outgoing) baryon and meson, and the coefficients $C_{i j ; V}^{(I, S, C)}$ denote the strength of the interaction in the different isospin $(I)$, strangeness $(S)$, charm $(C)$ sectors, and meson-baryon channels $(i, j)$. For the coupling constant $g$ we use the value 6.6, which reproduces the decay width of the $\rho$ meson [71]. The $s$-wave projection of the scattering kernel is easily obtained and, in the center-of-mass (c.m.) frame, it takes the analytical form

$$
\begin{aligned}
& V_{i j, l=0}^{(I, S, C)}\left(\vec{k}_{i}, \vec{k}_{j}\right) \\
& =N \frac{g^{2}}{8} \sum_{V \in[16]} C_{i j ; V}^{(I, S, C)}\left[\frac{2 \beta}{b}+\frac{\alpha b-\beta a}{b^{2}} \ln \left(\frac{a+b}{a-b}\right)\right],
\end{aligned}
$$

with $a, b, \alpha$, and $\beta$ being

$$
\begin{aligned}
a= & m_{i}^{2}+m_{j}^{2}-2 \omega_{i}\left(\left|\vec{k}_{i}\right|\right) \omega_{j}\left(\left|\vec{k}_{j}\right|\right)-m_{V}^{2} \quad b=2\left|\vec{k}_{i}\right|\left|\vec{k}_{j}\right| \\
\alpha= & \Omega_{i}\left(\left|\vec{k}_{i}\right|\right)+\Omega_{j}\left(\left|\vec{k}_{j}\right|\right)-M_{i}-M_{j} \\
& -\frac{m_{j}^{2}-m_{i}^{2}}{m_{V}^{2}}\left(\Omega_{j}\left(\left|\vec{k}_{j}\right|\right)-\Omega_{i}\left(\left|\vec{k}_{i}\right|\right)+M_{i}-M_{j}\right) \\
\beta= & \frac{\left|\vec{k}_{i}\right|\left|\vec{k}_{j}\right|}{\left(E_{i}\left(\left|\vec{k}_{i}\right|\right)+M_{i}\right)\left(E_{j}\left(\left|\vec{k}_{j}\right|\right)+M_{j}\right)}\left(\Omega_{i}\left(\left|\vec{k}_{i}\right|\right)+\Omega_{j}\left(\left|\vec{k}_{j}\right|\right)\right. \\
& \left.+M_{i}+M_{j}-\frac{m_{j}^{2}-m_{i}^{2}}{m_{V}^{2}}\left(\Omega_{j}\left(\left|\vec{k}_{j}\right|\right)-\Omega_{i}\left(\left|\vec{k}_{i}\right|\right)+M_{j}-M_{i}\right)\right),
\end{aligned}
$$

where $\vec{k}_{i}, \vec{k}_{j}$ are the initial and final relative momenta, $m_{i}$, $m_{j}, M_{i}, M_{j}$ are the masses of the incoming and outgoing mesons and baryons, $\omega_{i}\left(\left|\vec{k}_{i}\right|\right), \omega_{j}\left(\left|\vec{k}_{j}\right|\right), E_{i}\left(\left|\vec{k}_{i}\right|\right), E_{j}\left(\left|\vec{k}_{j}\right|\right)$ their corresponding energies, which have been taken to be their on-shell values, and $\Omega(|\vec{k}|)$ stands for the total energy $\omega(|\vec{k}|)+E(|\vec{k}|)$ of the meson-baryon pair. The factor $N=$ $\left[\left(E\left(\left|\vec{k}_{i}\right|\right)+M_{i}\right)\left(E\left(\left|\vec{k}_{j}\right|\right)+M_{j}\right) /\left(4 M_{i} M_{j}\right)\right]^{1 / 2}$ comes from the normalization of the Dirac spinors.

Once the scattering kernel has been constructed, it can be inserted in the $s$-wave projected Lipmann-Schwinger equation,

$$
\begin{aligned}
& T_{i j, l=0}^{(I, S, C)}\left(\vec{k}_{i}, \vec{k}_{j}, \sqrt{s}\right) \\
& =V_{i j, l=0}^{(I, S, C)}\left(\vec{k}_{i}, \vec{k}_{j}\right)+\sum_{m} \int \frac{d \vec{k}}{(2 \pi)^{3}} F(|\vec{k}|) V_{i m, l=0}^{(I, S, C)}\left(\vec{k}_{i}, \vec{k}\right) \\
& \quad \times J_{m}(\sqrt{s}, \vec{k}) T_{m j, l=0}^{(I, S, C)}\left(\vec{k}, \vec{k}_{j}, \sqrt{s}\right),
\end{aligned}
$$

where the loop function $J$ explicitly reads

$$
\begin{aligned}
J_{m}^{(I, S, C)}(\sqrt{s}, \vec{k})= & \frac{M_{m}}{2 E_{m}(|\vec{k}|) \omega_{m}(|\vec{k}|)} \\
& \times \frac{1}{\sqrt{s}-E_{m}(|\vec{k}|)-\omega_{m}(|\vec{k}|)+i \eta},
\end{aligned}
$$

and a dipole-type form factor $F(|\vec{k}|)$,

$$
F(|\vec{k}|)=\left(\frac{\Lambda^{2}}{\Lambda^{2}+|\vec{k}|^{2}}\right)^{2},
$$

has been introduced in order to regularize the integral. This form is typically adopted in studies of hadron-hadron interactions within the scheme of Lipmann-Schwinger-type 
TABLE I. Masses and widths of states that can be identified with well-established resonances in various sectors of $C=1$.

\begin{tabular}{|c|c|c|c|c|c|}
\hline \multirow[b]{2}{*}{$(I, S)$} & \multirow[b]{2}{*}{$\Lambda[\mathrm{MeV}]$} & \multicolumn{2}{|c|}{ Theory } & \multicolumn{2}{|c|}{ Experiment } \\
\hline & & $M_{R}[\mathrm{MeV}]$ & $\Gamma[\mathrm{MeV}]$ & $M_{R}[\mathrm{MeV}]$ & $\Gamma[\mathrm{MeV}]$ \\
\hline \multirow{2}{*}{$\begin{array}{l}(0,0) \\
\Lambda_{c} \\
(1,0)\end{array}$} & 903 & 2595 & 0.5 & $2595.4 \pm 0.6$ & $3.6_{1.3}^{+2.0}$ \\
\hline & & & & $2801_{-6}^{+4}$ & $75_{-17}^{+22}\left(\Sigma_{c}^{++}\right)$ \\
\hline \multirow[t]{2}{*}{$\Sigma_{c}$} & 1100 & 2792 & 16 & $2792_{-5}^{+14}$ & $62_{-40}^{+60}\left(\Sigma_{c}^{+}\right)$ \\
\hline & & & & $2802_{-7}^{+4}$ & $61_{-18}^{+28}\left(\Sigma_{c}^{0}\right)$ \\
\hline \multirow{2}{*}{$\begin{array}{l}(1 / 2,-1) \\
\Xi_{c}\end{array}$} & 980 & 2790 & 0.5 & $2791.8 \pm 3.3$ & $<12\left(\Xi_{c}^{0}\right)$ \\
\hline & 960 & 2970 & 5.1 & $2968.0 \pm 2.6$ & $20 \pm 7\left(\Xi_{c}^{0}\right)$ \\
\hline
\end{tabular}

equations in the light flavor sector [72]. The value of the cutoff $\Lambda$ is a free parameter of our model that is fitted to the existing data. In Table I we collect, from the resonances generated dynamically in Ref. [47] in various $C=1 \mathrm{sec}-$ tors, those states that, using a cutoff value of around $1 \mathrm{GeV}$, can be readily identified with observed resonances [71].

In order to associate a given enhancement of the scattering amplitude to a resonance, we look for a characteristic pole $z_{R}$ in the unphysical sheet of the complex energy plane. Our prescription of unphysical sheet is such that, whenever the real part of the complex energy crosses a meson-baryon threshold cut, the sign of the on-shell momentum is changed for this channel and for the already opened ones, as described in detail in Ref. [73]. If the pole $z_{R}$ lies not too far from the real axis, its value determines the Breit-Wigner mass $\left(M=\operatorname{Re} z_{R}\right)$ and width $(\Gamma=$ $2 \operatorname{Im} z_{R}$ ) of the resonance as seen from real energies. The couplings of the resonance into its various meson-baryon components are obtained from the residues of the scattering amplitude since, close to the pole, it can be parameterized in the form

$$
T_{i j, l=0}^{I, S, C}\left(\vec{k}_{i}, \vec{k}_{j}, z\right)=\frac{g_{i} g_{j}}{z-z_{R}} .
$$

Note that the value of the coupling constants of Eq. (12) depends on the particular momentum values chosen in the evaluation of the $T$-matrix element. However, this depen-

TABLE II. Coupling coefficients $g_{R B M}$ of the $\Lambda_{c}$ (2595) pole to the different meson-baryon (MB) channels.

\begin{tabular}{lc}
\hline \hline $\mathrm{MB}$ & $2595+i 0.25[\mathrm{MeV}]$ \\
\hline$\pi \Sigma_{c}$ & $0.06-i 0.31$ \\
$D N$ & $-0.13+i 11$ \\
$\eta \Lambda_{c}$ & $-0.005+i 0.42$ \\
$K \Xi_{c}$ & $-0.006+i 0.52$ \\
$K \Xi_{c}^{\prime}$ & $0.03-i 0.23$ \\
$D_{s} \Lambda$ & $-0.06+i 6$ \\
$\eta^{\prime} \Lambda_{c}$ & $-0.01+i 0.69$ \\
$\eta_{c} \Lambda_{c}$ & $0.02-i 2$ \\
$\bar{D} \Xi_{c c}$ & $0.007+i 0.05$ \\
\hline \hline
\end{tabular}

dence is very mild and we have obtained the couplings from the amplitudes at $\vec{k}_{i}=\vec{k}_{j}=0$. The values of the couplings of the resonances to the various charged meson-baryon states are listed in Tables II, III, and IV.

TABLE III. Coupling coefficients $g_{R B M}$ of the $\Sigma_{c}(2792)$ pole to the different meson-baryon (MB) channels.

\begin{tabular}{lc}
\hline \hline $\mathrm{MB}$ & $2792+i 8.16[\mathrm{MeV}]$ \\
\hline$\pi \Lambda_{c}$ & $0.19+i 0.53$ \\
$\pi \Sigma_{c}$ & $-0.28+i 0.12$ \\
$D N$ & $-0.60-i 3.6$ \\
$K \Xi_{c}$ & $0.31+i 0.28$ \\
$\eta \Sigma_{c}$ & $0.06-i 0.21$ \\
$K \Xi_{c}^{\prime}$ & $-0.13+i 0.24$ \\
$D_{s} \Sigma^{\prime}$ & $0.42+i 3.1$ \\
$\eta^{\prime} \Sigma_{c}$ & $-0.05-i 0.35$ \\
$\bar{D}_{c c} \Xi_{c c}$ & $0.22+i 0.28$ \\
$\eta_{c} \Sigma_{c}$ & $0.13+i 1$ \\
\hline \hline
\end{tabular}

TABLE IV. Coupling coefficients $g_{R B M}$ of the $\Xi_{c}(2790)$ and $\Xi_{c}(2970)$ poles to the different meson-baryon (MB) channels.

\begin{tabular}{lcc}
\hline \hline $\mathrm{MB}$ & $2790+i 0.25[\mathrm{MeV}]$ & $2970+i 2.5[\mathrm{MeV}]$ \\
\hline$\pi \Xi_{c}$ & $0.01+i 0.06$ & $0.008-i 0.37$ \\
$\pi \Xi_{c}^{\prime}$ & $0+i 0.002$ & $-0.25-i 0.08$ \\
$\bar{K} \Lambda_{c}$ & $-0.06-i 0.22$ & $0.1+i 0.17$ \\
$\bar{K} \Sigma_{c}$ & $-0.003-i 1.5$ & $-0.17+i 0.03$ \\
$D \Lambda$ & $0.08-i 2.1$ & $-0.21-i 3.2$ \\
$\eta \Xi_{c}$ & $-0.07-i 0.33$ & $0.08-i 0.02$ \\
$D \Sigma^{\prime}$ & $-0.03+i 12$. & $-0.07-i 1.76$ \\
$\eta \Xi_{c}^{\prime}$ & $-0.001-i 0.73$ & $-0.02-i 0.05$ \\
$K \Omega_{c}$ & $-0.003+i 0.08$ & $-0.21+i 0.15$ \\
$D_{s} \Xi^{\prime}$ & $-0.08+i 6.54$ & $0.18+i 2.8$ \\
$\eta^{\prime} \Xi_{c}$ & $0.005-i 0.76$ & $-0.007-i 0.08$ \\
$\eta^{\prime} \Xi_{c}^{\prime}$ & $0.004+i 0.16$ & $-0.01-i 0.31$ \\
$\bar{D}_{s} \Xi_{c c}$ & $0.04+i 0.44$ & $-0.02-i 0.006$ \\
$\bar{D} \Omega_{c c}$ & $-0.03+i 0.03$ & $-0.03+i 0.04$ \\
$\eta_{c} \Xi_{c}$ & $-0.007-i 1$ & $0.05+i 0.91$ \\
$\eta_{c} \Xi_{c}^{\prime}$ & $-0.01-i 0.46$ & $0.04+i 0.9$ \\
\hline \hline
\end{tabular}




\section{RADIATIVE DECAY CALCULATION}

The reaction we study is given by

$$
B^{*}\left(P, \chi_{i}\right) \rightarrow \gamma(K, \epsilon) B\left(Q, \chi_{f}\right),
$$

where $P, K$, and $Q$ are four-momenta which are related by $P=K+Q, \epsilon$ is the photon polarization, and $\chi_{i}$ and $\chi_{f}$ are the polarization of the initial and final spin- $\frac{1}{2}$ baryons $B^{*}$ and $B$, respectively.

For the evaluation of these radiative decays, we follow a gauge invariant scheme already used for noncharmed resonances [74-77]. As shown there, the mechanisms for the decay of the resonance $B^{*}$ into $\gamma B$ are given by the diagrams of Fig. 1, where the photon couples to the constituent mesons and baryons. The amplitude for the radiative decay has the structure

$$
-i \mathcal{M}=T_{\mu \nu} \epsilon^{\nu} \bar{\chi}_{f} \sigma^{\mu} \chi_{i}
$$

with $\sigma_{\mu}=(0, \vec{\sigma})$, where $\sigma_{i}$ are the usual Pauli matrices. For the tensor $T_{\mu \nu}$, there are five possible independent Lorentz structures that one can construct with the two independent four-momenta,

$T^{\mu \nu}=a g^{\mu \nu}+b P^{\mu} P^{\nu}+c P^{\mu} K^{\nu}+d K^{\mu} P^{\nu}+e K^{\mu} K^{\nu}$.

This expression is simplified by noting that, due to the Lorentz condition $\epsilon_{\nu} K^{\nu}=0$, the terms with the coefficients $c$ and $e$ in Eq. (11) will not contribute to the radiative decay amplitude of Eq. (10). Moreover, gauge invariance imposes $T_{\mu \nu} K^{\nu}=0$ and we obtain

$$
(a+d(P \cdot K)) K^{\mu}+b(P \cdot K) P^{\mu}=0,
$$

from which we conclude that $b=0$, while $a$ and $d$ are related through $a=-d(P \cdot K)$. The expression for the amplitude can be further simplified if we work in the rest frame of the decaying baryon $(\vec{P}=\overrightarrow{0})$ and take the Coulomb gauge $\left(\epsilon^{0}=0\right)$. In this way only the $a$ term contributes to the amplitude. Moreover, the $a$ coefficient

(a)

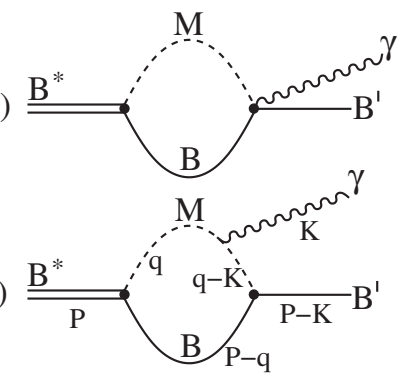

(c)

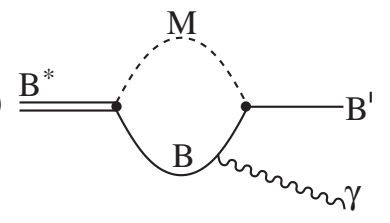

FIG. 1. Diagrams needed for the evaluation of the radiative decay of dynamically generated baryons. is more easily calculated through the $d$ coefficient, using the above mentioned relation, $a=-d(P \cdot K)$. This is so because, as we will show, the integral for evaluating the $d$ coefficient converges and only the diagrams of Figs. 1(b) and $1(\mathrm{c})$ contribute to it.

The amplitude for diagram 1(b) is given by

$$
\begin{aligned}
-i \mathcal{M}= & -i g_{R B M} g_{B B M} g_{\gamma M M} \int \frac{d^{4} q}{(2 \pi)^{4}} \frac{1}{(q-K)^{2}-m_{M}^{2}} \\
& \times \frac{1}{q^{2}-m_{M}^{2}} \frac{2 m_{B}}{(P-q)^{2}-m_{B}^{2}} \\
& \times \bar{\chi}_{f}(q-K) \cdot \sigma \chi_{i}(2 q-K) \cdot \epsilon,
\end{aligned}
$$

where $g_{R B M}$ is the coupling of the resonance to its meson and baryon constituents, $g_{B B M}$ is the coupling of the baryonic current to a meson, $g_{\gamma M M}$ is the coupling of the meson to a photon, and $m_{M}$ and $m_{B}$ are masses of the meson and baryon in the loop, respectively. Using the Feynman parameterization

$\frac{1}{a b c}=2 \int_{0}^{1} d x \int_{0}^{1-x} d y \frac{1}{(x(a-b)+y(c-b)+b)^{3}}$,

and the identity

$$
\int d^{4} q^{\prime} \frac{1}{\left(q^{\prime 2}+s+i \epsilon\right)^{3}}=\frac{i \pi^{2}}{2} \frac{1}{s+i \epsilon},
$$

together with the relation between the $a$ and $d$ terms of the amplitude, one finds the following contribution of diagram 1(b) to the $a$ term:

$$
\begin{aligned}
&-i \mathcal{M}_{(\mathrm{b})}^{a}= g_{R B M} g_{B B M} g_{\gamma M M} \frac{2 m_{B}}{(4 \pi)^{2}} \\
& \times \int_{0}^{1} d x \int_{0}^{1-x} d y \frac{x(y-1)}{s+i \epsilon} 2 P \cdot K \\
& s=x\left(P^{2}(1-x)-m_{B}^{2}+m_{M}^{2}-2 y P \cdot K\right)-m_{M}^{2} .
\end{aligned}
$$

Analogously, one can calculate the contribution coming from the diagram in Fig. 1(c) as

$$
\begin{aligned}
&-i \mathcal{M}_{(\mathrm{c})}^{a}= g_{R B M} g_{B B M} g_{\gamma B B} \frac{2 m_{B}}{(4 \pi)^{2}} \\
& \times \int_{0}^{1} d x \int_{0}^{1-x} d y \frac{-y x}{s+i \epsilon} 2 P \cdot K \\
& s=x\left(P^{2}(1-x)-m_{M}^{2}+m_{B}^{2}-2 y P \cdot K\right)-m_{B}^{2} .
\end{aligned}
$$

The values of the couplings $g_{R B M}$, relating the resonance to its constituents, are given, in the isospin basis, in Tables II, III, and IV of the previous section. The couplings of the mesons to the photon, $g_{\gamma M M}$, and those of the baryons to the photon, $g_{\gamma B B}$, are $e Q$, where $Q$ is the electric charge $(-1,0,1$, or 2$)$ of the meson or the baryon which emits the photon. Finally, the coupling of the mesons to the ground state baryons is given by 


$$
g_{B B M}=\alpha_{i} \frac{D+F}{2 f}+\beta_{i} \frac{D-F}{2 f},
$$

where $D+F=1.26, D-F=0.33$ [78]. We have taken $f=1.15 f_{M}$ with $f_{M}=f_{\pi}=93 \mathrm{MeV}$ for light mesons and $f_{M}=f_{D}=165 \mathrm{MeV}$ for heavy mesons. The coefficients $\alpha_{i}$ and $\beta_{i}$, which depend on the SU(4) flavor structure of the interaction, have been obtained from the Lagrangian for the interaction of the ground state spin $\frac{1^{+}}{2}$ baryons and the pseudoscalar mesons, which is constructed in the following.

Our prescription of field definitions for the baryons and mesons is that of Ref. [43]. The baryons are represented by a three-index tensor $\mathcal{B}^{i j k}$, antisymmetric in the first two indices, where $i, j$, and $k$ run from 1 to 4 ,

$\mathcal{B}^{121}=p, \quad \mathcal{B}^{122}=n, \quad \mathcal{B}^{132}=\frac{1}{\sqrt{2}} \Sigma^{0}-\frac{1}{\sqrt{6}} \Lambda$,

$\mathcal{B}^{124}=\frac{2}{\sqrt{6}} \Lambda_{c}, \quad \mathcal{B}^{141}=-\Sigma_{c}^{++}$,

$\mathcal{B}^{142}=\frac{1}{\sqrt{2}} \Sigma_{c}^{+}+\frac{1}{\sqrt{6}} \Lambda_{c}, \quad \mathcal{B}^{143}=\frac{1}{\sqrt{2}} \Xi_{c}^{\prime+}-\frac{1}{\sqrt{6}} \Xi_{c}^{+}$,

$\mathcal{B}^{144}=\Xi_{c c}^{++}, \quad \mathcal{B}^{213}=\frac{2}{\sqrt{6}} \Lambda$,

$\mathcal{B}^{231}=\frac{1}{\sqrt{2}} \Sigma^{0}+\frac{1}{\sqrt{6}} \Lambda, \quad \mathcal{B}^{232}=\Sigma^{-}$,

$\mathcal{B}^{233}=\Xi^{-}, \quad \mathcal{B}^{234}=\frac{2}{\sqrt{6}} \Xi_{c}^{0}$,

$\mathcal{B}^{241}=\frac{1}{\sqrt{2}} \Sigma_{c}^{+}-\frac{1}{\sqrt{6}} \Lambda_{c}, \quad \mathcal{B}^{242}=\Sigma_{c}^{0}$,

$\mathcal{B}^{243}=\frac{1}{\sqrt{2}} \Xi_{c}^{\prime 0}+\frac{1}{\sqrt{6}} \Xi_{c}^{0}, \quad \mathcal{B}^{244}=-\Xi_{c c}^{+}$,

$\mathcal{B}^{311}=\Sigma^{+}, \quad \mathcal{B}^{313}=\Xi^{0}, \quad \mathcal{B}^{314}=\frac{2}{\sqrt{6}} \Xi_{c}^{+}$,

$\mathcal{B}^{341}=\frac{1}{\sqrt{2}} \Xi_{c}^{\prime+}+\frac{1}{\sqrt{6}} \Xi_{c}^{+}, \quad \mathcal{B}^{342}=\frac{1}{\sqrt{2}} \Xi_{c}^{\prime 0}-\frac{1}{\sqrt{6}} \Xi_{c}^{0}$,

$\mathcal{B}^{343}=\Omega_{c}, \quad \mathcal{B}^{344}=\Omega_{c c}$

The pseudoscalar meson field is represented by a matrix,

$\Phi=\left(\begin{array}{cccc}\frac{\pi^{0}}{\sqrt{2}}+\frac{\eta_{8}}{\sqrt{6}}+\frac{\eta_{15}}{\sqrt{12}} & \pi^{+} & K^{+} & \bar{D}^{0} \\ \pi^{-} & \frac{\pi^{0}}{\sqrt{2}}+\frac{\eta_{8}}{\sqrt{6}}+\frac{\eta_{15}}{\sqrt{12}} & K^{0} & -D^{-} \\ K^{-} & \bar{K}^{0} & \frac{-2 \eta_{8}}{\sqrt{6}}+\frac{\eta_{15}}{\sqrt{12}} & D_{s}^{-} \\ D^{0} & -D^{+} & D_{s}^{+} & \frac{-3 \eta_{15}}{\sqrt{12}}\end{array}\right)$.
The Lagrangian for the $B B M$ interaction reads

$$
\begin{aligned}
\mathcal{L}_{B B M}= & \frac{\sqrt{2}}{4 f} \sum_{i, j, k, l=1}^{4} \overline{\mathcal{B}}_{i j k} \gamma^{\mu}\left((D+F) \partial_{\mu} \Phi_{k l} \mathcal{B}_{i j l}\right. \\
& \left.-2(D-F) \partial_{\mu} \Phi_{j l} \mathcal{B}_{i l k}\right) .
\end{aligned}
$$

Note that the physical $\eta, \eta^{\prime}$, and $\eta_{c}$ fields are related to the mathematical $\eta_{8}$ and $\eta_{15}$ fields, belonging to the pseudoscalar meson 15-plet, and the singlet $\eta_{1}$ field. The mixture is given by

$$
\left(\begin{array}{c}
\eta_{1} \\
\eta_{8} \\
\eta_{15}
\end{array}\right)=\left(\begin{array}{ccc}
\frac{1}{2 \sqrt{3}} & \sqrt{\frac{2}{3}} & \frac{1}{2} \\
\frac{2 \sqrt{2}}{3} & -\frac{1}{3} & 0 \\
\frac{1}{6} & \frac{\sqrt{2}}{3} & -\frac{\sqrt{3}}{2}
\end{array}\right)\left(\begin{array}{c}
\eta \\
\eta^{\prime} \\
\eta_{c}
\end{array}\right) .
$$

Therefore, in order to obtain a Lagrangian for the physical fields, we need to add to Eq. (23) an interaction of the baryon current with the singlet field $\eta_{1}$,

$$
\begin{aligned}
\mathcal{L}_{B B M}^{\prime}= & \mathcal{L}_{B B M}+\frac{\sqrt{2}}{4 f}\left(D \delta_{D}+F \delta_{F}\right) \frac{1}{2} \\
& \times \sum_{i, j, k=1}^{4} \overline{\mathcal{B}}_{i j k} \gamma^{\mu} \mathcal{B}_{i j k} \partial_{\mu} \eta_{1},
\end{aligned}
$$

where the coefficients $\delta_{D}=-\frac{1}{\sqrt{2}}$ and $\delta_{F}=\frac{3}{\sqrt{2}}$ are obtained by consistently imposing that processes where the $\eta_{c}$ meson couples to light baryons $(N, \Lambda, \Sigma$, or $\Xi)$ should vanish.

The complete Lagrangian $\mathcal{L}_{B B M}^{\prime}$ of Eq. (25) allows us to determine all coupling constants $g_{B B M}$, which we write in the form of Eq. (20). The specific values of the $\alpha_{i}$ and $\beta_{i}$ coefficients for all the transitions needed in the different radiative decays are collected in Tables VI, VII, VIII, IX, and $\mathrm{X}$ of the Appendix.

With all the required ingredients having been established, one can finally obtain the radiative decay width from

$$
\Gamma=\frac{1}{\pi}\left|\sum_{i} \mathcal{M}_{i}^{a}\right|^{2} E_{\gamma} \frac{m_{B}}{m_{B^{*}}},
$$

where $E_{\gamma}$ is the photon energy, $\mathcal{M}_{i}^{a}=\mathcal{M}_{(b)}^{a}+\mathcal{M}_{(c)}^{a}$, and the sum runs over all contributing intermediate $M B$ channels.

\section{RESULTS}

We evaluate the following radiative decays:

$$
\Lambda_{c}(2595) \rightarrow \Lambda_{c} \gamma
$$




$$
\begin{gathered}
\Lambda_{c}(2595) \rightarrow \Sigma_{c}^{+} \gamma \\
\Sigma_{c}^{+}(2792) \rightarrow \Lambda_{c} \gamma \\
\Sigma_{c}^{++}(2792) \rightarrow \Sigma_{c}^{++} \gamma \\
\Sigma_{c}^{+}(2792) \rightarrow \Sigma_{c}^{+} \gamma \\
\Sigma_{c}^{0}(2792) \rightarrow \Sigma_{c}^{0} \gamma \\
\Xi_{c}^{+}(2790) \rightarrow \Xi_{c}^{+} \gamma \\
\Xi_{c}^{0}(2790) \rightarrow \Xi_{c}^{0} \gamma \\
\Xi_{c}^{+}(2790) \rightarrow \Xi_{c}^{\prime+} \gamma \\
\Xi_{c}^{0}(2790) \rightarrow \Xi_{c}^{\prime 0} \gamma \\
\Xi_{c}^{+}(2970) \rightarrow \Xi_{c}^{+} \gamma \\
\Xi_{c}^{0}(2970) \rightarrow \Xi_{c}^{0} \gamma \\
\Xi_{c}^{+}(2970) \rightarrow \Xi_{c}^{\prime+} \gamma \\
\Xi_{c}^{0}(2970) \rightarrow \Xi_{c}^{\prime 0} \gamma,
\end{gathered}
$$

for $\Lambda_{c}(2595), \Sigma_{c}(2792), \Xi_{c}(2790)$, and $\Xi_{c}(2970)$, which are the resonances that, according to the models of Ref. [40-47], can plausibly be interpreted as dynamically generated meson-baryon molecules.
The theoretical decay widths obtained from the imaginary part of the pole position for these dynamically generated states is usually smaller than the experimental observed decay widths. The model considers only meson-baryon channels where the meson is a pseudoscalar coupled in $s$-wave with a $J^{P}=\frac{1^{+}}{2}$ baryon. In the case of the heavier resonances, the model is probably missing possible three-body decays of the resonance, or channels where the meson and baryon have different $J^{P}$ quantum numbers and this should partially explain the small widths found for the theoretical resonances. In any case one must assume some uncertainties for the couplings listed in Tables II, III, and IV.

We estimate the uncertainties in the radiative decay widths assuming a $20 \%$ uncertainty in the $g_{R B M}$ couplings. Following the method of Ref. [64], we generate, for each resonance, 30 sets of random coupling values within $20 \%$ of those listed in Tables II, III, and IV, and calculate the radiative decay for each set. Our final result is the average of the radiative decay widths obtained with the random sets, and the associated uncertainty is given by the standard deviation from the average.

\begin{tabular}{|c|c|c|c|c|c|c|c|}
\hline Channel & $\Gamma_{\Lambda_{c} \gamma}[\mathrm{keV}]$ & {$[55][\mathrm{keV}]$} & {$[61][\mathrm{keV}]$} & {$[62][\mathrm{keV}]$} & $\Gamma_{\Sigma_{c}^{+} \gamma}[\mathrm{keV}]$ & {$[55][\mathrm{keV}]$} & {$[61][\mathrm{keV}]$} \\
\hline$\pi^{+} \Sigma_{c}^{0}$ & $1.2(+)$ & & & & $0.3(-)$ & & \\
\hline$\pi^{0} \Sigma_{c}^{+}$ & $<0.1(+)$ & & & & 0 & & \\
\hline$\pi^{-} \Sigma_{c}^{++}$ & $0.7(-)$ & & & & $0.2(-)$ & & \\
\hline$D^{0} p$ & $9.1(+)$ & & & & $0.1(+)$ & & \\
\hline$D^{+} n$ & $83.4(+)$ & & & & $0.8(-)$ & & \\
\hline$\eta \Lambda_{c}$ & $<0.1(+)$ & & & & 0 & & \\
\hline$K^{+} \Xi_{c}^{0}$ & $<0.1(+)$ & & & & $<0.1(+)$ & & \\
\hline$K^{0} \Xi_{c}^{+}$ & $<0.1(+)$ & & & & $<0.1(-)$ & & \\
\hline$K^{+} \Xi_{c}^{\prime \prime 0}$ & $<0.1(+)$ & & & & $<0.1(-)$ & & \\
\hline$K^{0} \Xi_{c}^{\prime+}$ & $<0.1(+)$ & & & & $<0.1(+)$ & & \\
\hline$D_{s}^{+} \Lambda$ & $13.5(+)$ & & & & 0 & & \\
\hline$\eta^{\prime} \Lambda_{c}$ & $<0.1(+)$ & & & & 0 & & \\
\hline$\eta_{c} \Lambda_{c}$ & $<0.1(+)$ & & & & 0 & & \\
\hline $\bar{D}^{0} \Xi_{c c}^{+}$ & $<0.1(+)$ & & & & $<0.1(-)$ & & \\
\hline$D^{-} \Xi_{c c}^{++}$ & $<0.1(-)$ & & & & $<0.1(-)$ & & \\
\hline Total & $274.3 \pm 52.0$ & 115 & 36 & 16 & $2.1 \pm 0.4$ & 77 & 11 \\
\hline
\end{tabular}

We start showing the results for the $\Lambda_{c}(2595)$, which is the analog in the charm sector of the $\Lambda(1405)$ in the strange sector and, therefore, is a good candidate for being a meson-baryon molecular state. The radiative decay widths are

$$
\begin{gathered}
\Gamma_{\Lambda_{c}(2595) \rightarrow \Lambda_{c} \gamma}=(274.3 \pm 52.0) \mathrm{keV} \\
\Gamma_{\Lambda_{c}(2595) \rightarrow \Sigma_{c}^{+} \gamma}=(2.1 \pm 0.4) \mathrm{keV} .
\end{gathered}
$$

These values are also collected in Table V, where the contribution to the width coming from each intermediate

TABLE V. Results for the radiative decay of the $\Lambda_{c}(2595)$ compared with other theoretical approaches. The sign in brackets indicates the sign of the amplitude, so one can know when the interference between the channels is constructive or destructive. 
channel is also shown, together with the sign, $(+)$ or $(-)$, of the corresponding amplitude. This allows one to analyze the constructive or destructive character of the interferences between the various channels. First of all, we note the tremendous difference in size, of 2 orders of magnitude, between the radiative decay rate of the $\Lambda_{c}(2595)$ into $\Lambda_{c} \gamma$ and $\Sigma_{c}^{+} \gamma$ states. To understand the origin of this difference we focus on the most important contribution, which corresponds to the $D^{+} n$ intermediate state in both cases. First of all, the ratio between the couplings $D^{+} n \rightarrow$ $\Lambda_{c}$ and $D^{+} n \rightarrow \Sigma_{c}^{+}$is 3.8 , as can be inferred from the $\alpha$ and $\beta$ coefficients of Table VI. Moreover, the different kinematical variables of the two processes produce a $D^{+} n$ loop which is, for $\Lambda_{c} \gamma$ decay, a factor 2 larger than for $\Sigma_{c}^{+} \gamma$ decay. The square of these two factors, together with the ratio of photon energies, which are $E_{\gamma}=290 \mathrm{MeV}$ for $\Lambda_{c} \gamma$ and $E_{\gamma}=138 \mathrm{MeV}$ for $\Sigma_{c}^{+} \gamma$, explain the factor 100 difference between the $D^{+} n$ contributions to both decays. We also note that there are constructive interferences between the most important contributions $\left(D^{0} p, D^{+} n, D_{s}^{+} \Lambda\right)$ to $\Lambda_{c} \gamma$ decay, which enhance even further this rate compared to the $\Sigma_{c}^{+} \gamma$ one.

In Table $\mathrm{V}$ we also compare our results to those obtained by other calculations performed within the relativistic quark model [55], using light-cone QCD sum rules [61], or adopting a bound $D^{*} N$ picture for the $\Lambda_{c}(2595)$ [62]. We observe a large diversity of results. Note also that the 2 orders of magnitude difference between the radiative decays into $\Lambda_{c} \gamma$ or $\Sigma_{c} \gamma$ states found in the present work is not obtained by any of the other models displayed in Table V, nor by the HHCPT results of Ref. [60], which estimated partial widths of the same order of magnitude. Obviously, the tremendous differences between models calls for a measurement of these decay modes which could bring essential information about the nature of the $\Lambda_{c}(2595)$.

The radiative decays of the other resonances, for which there is no experimental observation nor other theoretical predictions, are

$$
\begin{gathered}
\Gamma_{\Sigma_{c}^{++}(2792) \rightarrow \Sigma_{c}^{++} \gamma}=(51.0 \pm 9.1) \mathrm{keV} \\
\Gamma_{\Sigma_{c}^{+}(2792) \rightarrow \Sigma_{c}^{+} \gamma}=(28.4 \pm 3.3) \mathrm{keV} \\
\Gamma_{\Sigma_{c}^{0}(2792) \rightarrow \Sigma_{c}^{0} \gamma}=(9.1 \pm 1.5) \mathrm{keV} \\
\Gamma_{\Sigma_{c}^{+}(2792) \rightarrow \Lambda_{c} \gamma}=(33.5 \pm 8.8) \mathrm{keV} \\
\Gamma_{\Xi_{c}^{+}(2790) \rightarrow \Xi_{c}^{+} \gamma}=(249.6 \pm 41.9) \mathrm{keV} \\
\Gamma_{\Xi_{c}^{0}(2790) \rightarrow \Xi_{c}^{0} \gamma}=(119.3 \pm 21.7) \mathrm{keV}
\end{gathered}
$$

$$
\begin{gathered}
\Gamma_{\Xi_{c}^{+}(2790) \rightarrow \Xi_{c}^{\prime+} \gamma}=(0.8 \pm 0.5) \mathrm{keV} \\
\Gamma_{\Xi_{c}^{0}(2790) \rightarrow \Xi_{c}^{\prime 0} \gamma}=(1.3 \pm 0.4) \mathrm{keV} \\
\Gamma_{\Xi_{c}^{+}(2970) \rightarrow \Xi_{c}^{+} \gamma}=(56.4 \pm 19.2) \mathrm{keV} \\
\Gamma_{\Xi_{c}^{0}(2970) \rightarrow \Xi_{c}^{0} \gamma}=(2.5 \pm 1.7) \mathrm{keV} \\
\Gamma_{\Xi_{c}^{+}(2970) \rightarrow \Xi_{c}^{\prime+} \gamma}=(40.1 \pm 6.9) \mathrm{keV} \\
\Gamma_{\Xi_{c}^{0}(2970) \rightarrow \Xi_{c}^{\prime 0} \gamma}=(9.2 \pm 1.0) \mathrm{keV} .
\end{gathered}
$$

It would also be interesting to see whether our predictions, based in a molecular picture for these resonances, are also very different from those obtained in quark model theoretical approaches.

We finally note that the experimental strong decay width of the $\Lambda_{c}(2595)$ is $3.6 \mathrm{MeV}$, the $80 \%$ of which, $2.9 \mathrm{MeV}$, corresponds to the decay into $\pi \Sigma_{c}$ states. Our value for the coupling of this resonance to $\pi \Sigma_{c},\left|g_{\pi \Sigma_{c}}\right|=0.32$, gives a $\pi \Sigma_{c}$ width of $0.5 \mathrm{MeV}$ while the experimental value would be reproduced by taking $\left|g_{\pi \Sigma_{c}}\right|=0.76$. Calculating the radiative decay of the $\Lambda_{c}(2595)$ with this new absolute value for the coupling produces

$$
\begin{gathered}
\Gamma_{\Lambda_{c}(2595) \rightarrow \Lambda_{c} \gamma}=(289.1 \pm 53.3) \mathrm{keV} \\
\Gamma_{\Lambda_{c}(2595) \rightarrow \Sigma_{c}^{+} \gamma}=(7.9 \pm 1.5) \mathrm{keV} .
\end{gathered}
$$

We observe that the changes to the decay width into $\Lambda_{c} \gamma$ states fall within the estimated theoretical uncertainties, while the changes for the decay into $\Sigma_{c}^{+} \gamma$ states are larger. The reason is that, as seen in Table $\mathrm{V}$, the $\Gamma_{\Lambda_{c}(2595) \rightarrow \Lambda_{c} \gamma}$ width is essentially dominated by $D N$ contributions, while the intermediate $\pi \Sigma_{c}$ states give a more significant relative contribution to the $\Gamma_{\Sigma_{c}(2595) \rightarrow \Sigma_{c}^{+} \gamma}$ width. In any case, the decay of the $\Lambda_{c}(2595)$ into $\Lambda_{c} \gamma$ is still very large compared to its decay into $\Sigma_{c} \gamma$, a result that distinguishes the present work from other approaches in the literature.

Correcting manually the couplings of the other resonances, for which there is some experimental data on their strong decays, has an even smaller effect on the widths than in the case of the $\Lambda_{c}(2595)$.

\section{CONCLUSIONS AND OVERVIEW}

We have studied the radiative decay width of charmed baryon resonances, which have been obtained as poles in the $T$-matrix of a coupled-channels dynamical model and, therefore, they are interpreted as composite meson-baryon molecular states. 
We have obtained a sizable value for the one-photon radiative width of the $\Lambda_{c}(2595)$. This resonance decays radiatively mostly into $\Lambda_{c} \gamma$ states, with a partial rate of close to $300 \mathrm{keV}$, while the decay width to $\Sigma_{c} \gamma$ is smaller that $10 \mathrm{keV}$. Our results are very different, in size and distribution among decay channels, to what is found by other approaches in the literature.

We have also presented predictions for the radiative decay of other excited charmed baryons. The radiative decay widths of the $\Sigma_{c}(2800)$ and $\Xi_{c}(2970)$ resonances are found to be relatively small, of the order of a few tenths of $\mathrm{keV}$. However, the transitions $\Xi_{c}(2790)^{+} \rightarrow \Xi_{c}^{+} \gamma$ and $\Xi_{c}(2790)^{0} \rightarrow \Xi_{c}^{0} \gamma$ are substantially larger, worth exploring experimentally.

The sizable value of some widths, especially those of the $\Lambda_{c}(2595)$ and the $\Xi_{c}(2790)$ resonances, makes the study of these radiative reactions a very useful tool to obtain information about the characteristics of these charmed baryons.

TABLE VI. The $\alpha$ and $\beta$ coefficients for the meson-baryon $(\mathrm{MB})$ channels involved in the radiative decay of resonances into $\Lambda_{c}$ and $\Sigma_{c}^{+}$.

\begin{tabular}{|c|c|c|c|c|}
\hline \multirow[b]{2}{*}{ MB } & \multicolumn{2}{|c|}{$\Lambda_{c}$} & \multicolumn{2}{|c|}{$\Sigma_{c}^{+}$} \\
\hline & $\alpha$ & $\beta$ & $\alpha$ & $\beta$ \\
\hline$\pi^{+} \Sigma_{c}^{0}$ & $-\frac{1}{\sqrt{3}}$ & $-\frac{1}{\sqrt{3}}$ & 1 & -1 \\
\hline$\pi^{0} \Sigma_{c}^{+}$ & $-\frac{1}{\sqrt{3}}$ & $-\frac{1}{\sqrt{3}}$ & 0 & 0 \\
\hline$\pi^{-} \Sigma_{c}^{++}$ & $-\frac{1}{\sqrt{3}}$ & $-\frac{1}{\sqrt{3}}$ & -1 & 1 \\
\hline$D^{0} p$ & $\frac{2}{\sqrt{3}}$ & $-\frac{1}{\sqrt{3}}$ & 0 & 1 \\
\hline$D^{+} n$ & $-\frac{2}{\sqrt{3}}$ & $\frac{1}{\sqrt{3}}$ & 0 & 1 \\
\hline$\eta \Lambda_{c}$ & $\frac{\sqrt{2}}{3 \sqrt{3}}$ & $\frac{5 \sqrt{2}}{3 \sqrt{3}}$ & 0 & 0 \\
\hline$K^{+} \Xi_{c}^{0}$ & $-\frac{1}{3 \sqrt{2}}$ & $\frac{5}{3 \sqrt{2}}$ & $\frac{1}{\sqrt{6}}$ & $\frac{1}{\sqrt{6}}$ \\
\hline$K^{0} \Xi_{c}^{+}$ & $-\frac{1}{3 \sqrt{2}}$ & $\frac{5}{3 \sqrt{2}}$ & $-\frac{1}{\sqrt{6}}$ & $-\frac{1}{\sqrt{6}}$ \\
\hline$K^{+} \Xi_{c}^{\prime 0}$ & $-\frac{1}{\sqrt{6}}$ & $-\frac{1}{\sqrt{6}}$ & $\frac{1}{\sqrt{2}}$ & $-\frac{1}{\sqrt{2}}$ \\
\hline$K^{0} \Xi_{c}^{\prime+}$ & $\frac{1}{\sqrt{6}}$ & $\frac{1}{\sqrt{6}}$ & $\frac{1}{\sqrt{2}}$ & $-\frac{1}{\sqrt{2}}$ \\
\hline$D_{s}^{+} \Lambda$ & $-\frac{2 \sqrt{2}}{3}$ & $\frac{\sqrt{2}}{3}$ & 0 & 0 \\
\hline$\eta^{\prime} \Lambda_{c}$ & $\frac{1}{3 \sqrt{3}}$ & $-\frac{5}{3 \sqrt{3}}$ & 0 & 0 \\
\hline$\eta_{c} \Lambda_{c}$ & $\frac{2 \sqrt{2}}{3}$ & $-\frac{\sqrt{2}}{3}$ & 0 & 0 \\
\hline $\bar{D}^{0} \Xi_{c c}^{+}$ & $\frac{1}{\sqrt{3}}$ & $-\frac{2}{\sqrt{3}}$ & -1 & 0 \\
\hline$D^{-} \Xi_{c c}^{++}$ & $-\frac{1}{\sqrt{3}}$ & $\frac{2}{\sqrt{3}}$ & -1 & 0 \\
\hline$\pi^{0} \Lambda_{c}$ & 0 & 0 & $-\frac{1}{\sqrt{3}}$ & $-\frac{1}{\sqrt{3}}$ \\
\hline$\eta \Sigma_{c}^{+}$ & 0 & 0 & $\sqrt{\frac{2}{3}}$ & $-\sqrt{\frac{2}{3}}$ \\
\hline$D_{s}^{+} \Sigma^{0}$ & 0 & 0 & 0 & $-\sqrt{2}$ \\
\hline$\eta^{\prime} \Sigma_{c}^{+}$ & 0 & 0 & $\frac{1}{\sqrt{3}}$ & $-\frac{1}{\sqrt{3}}$ \\
\hline$\eta_{c} \Sigma_{c}^{+}$ & 0 & 0 & 0 & $-\sqrt{2}$ \\
\hline
\end{tabular}

\section{ACKNOWLEDGMENTS}

The authors would like thank Isaac Vidaña for useful discussions. This work is partly supported by the EU Contract No. MRTN-CT-2006-035482 (FLAVIAnet), by the Contract No. FIS2008-01661 from MICINN (Spain), and by the Generalitat de Catalunya Contract No. 2009SGR-1289. We acknowledge the support of the European Community-Research Infrastructure Integrating Activity "Study of Strongly Interacting Matter" (HadronPhysics2, Grant Agreement No. 227431) under the Seventh Framework Programme of EU.

\section{APPENDIX: BARYON-BARYON-MESON COUPLING CONSTANTS $g_{B B M}$}

This Appendix gives the values of the coefficients $\alpha_{i}$ and $\beta_{i}$ that define, according to Eq. (20), the $B B M$ coupling constants $g_{B B M}$ needed in the diagrams that determine the various radiative decays (Tables VI, VII, VIII, IX, and $\mathrm{X})$.

TABLE VII. The $\alpha$ and $\beta$ coefficients for the meson-baryon (MB) channels involved in the radiative decay of resonances into $\Xi_{c}^{+}$and $\Xi_{c}^{\prime+}$.

\begin{tabular}{|c|c|c|c|c|}
\hline \multirow[b]{2}{*}{ MB } & \multicolumn{2}{|c|}{$\Xi_{c}^{+}$} & \multicolumn{2}{|c|}{$\Xi_{c}^{\prime+}$} \\
\hline & $\alpha$ & $\beta$ & $\alpha$ & $\beta$ \\
\hline$\pi^{+} \Xi_{c}^{0}$ & $-\frac{1}{3 \sqrt{2}}$ & $\frac{5}{3 \sqrt{2}}$ & $-\frac{1}{\sqrt{6}}$ & $-\frac{1}{\sqrt{6}}$ \\
\hline$\pi^{0} \Xi_{c}^{+}$ & $\frac{1}{6}$ & $-\frac{5}{6}$ & $\frac{1}{2 \sqrt{3}}$ & $\frac{1}{2 \sqrt{3}}$ \\
\hline$\pi^{+} \Xi_{c}^{\prime 0}$ & $\frac{1}{\sqrt{6}}$ & $\frac{1}{\sqrt{6}}$ & $\frac{1}{\sqrt{2}}$ & $-\frac{1}{\sqrt{2}}$ \\
\hline$\pi^{0} \Xi_{c}^{\prime+}$ & $\frac{1}{2 \sqrt{3}}$ & $\frac{1}{2 \sqrt{3}}$ & $\frac{1}{2}$ & $-\frac{1}{2}$ \\
\hline $\bar{K}^{0} \Lambda_{c}$ & $\frac{1}{3 \sqrt{2}}$ & $-\frac{5}{3 \sqrt{2}}$ & $\frac{1}{\sqrt{6}}$ & $\frac{1}{\sqrt{6}}$ \\
\hline $\bar{K}^{0} \Sigma_{c}^{+}$ & $-\frac{1}{\sqrt{6}}$ & $-\frac{1}{\sqrt{6}}$ & $\frac{1}{\sqrt{2}}$ & $-\frac{1}{\sqrt{2}}$ \\
\hline$K^{-} \Sigma_{c}^{++}$ & $\frac{1}{\sqrt{3}}$ & $\frac{1}{\sqrt{3}}$ & -1 & 1 \\
\hline$D^{+} \Lambda$ & $-\frac{\sqrt{2}}{3}$ & $\frac{1}{3 \sqrt{2}}$ & 0 & $\sqrt{\frac{3}{2}}$ \\
\hline$\eta \Xi_{c}^{+}$ & 0 & 0 & $\frac{\sqrt{2}}{3}$ & $\frac{\sqrt{2}}{3}$ \\
\hline$D^{+} \Sigma^{0}$ & $\sqrt{\frac{2}{3}}$ & $-\frac{1}{\sqrt{6}}$ & 0 & $-\frac{1}{\sqrt{2}}$ \\
\hline$D^{0} \Sigma^{+}$ & $\frac{2}{\sqrt{3}}$ & $-\frac{1}{\sqrt{3}}$ & 0 & -1 \\
\hline$\eta \Xi_{c}^{\prime+}$ & $\frac{\sqrt{2}}{3}$ & $\frac{\sqrt{2}}{3}$ & 0 & 0 \\
\hline$K^{+} \Omega_{c}$ & $\frac{1}{\sqrt{3}}$ & $\frac{1}{\sqrt{3}}$ & 1 & -1 \\
\hline$D_{s}^{+} \Xi^{0}$ & $\frac{2}{\sqrt{3}}$ & $-\frac{1}{\sqrt{3}}$ & 0 & 1 \\
\hline$\eta^{\prime} \Xi_{c}^{+}$ & $\frac{1}{2 \sqrt{3}}$ & $\frac{5}{2 \sqrt{3}}$ & $-\frac{1}{6}$ & $-\frac{1}{6}$ \\
\hline$\eta^{\prime} \Xi_{c}^{\prime+}$ & $-\frac{1}{6}$ & $-\frac{1}{6}$ & $\frac{\sqrt{3}}{2}$ & $-\frac{\sqrt{3}}{2}$ \\
\hline$D_{s}^{-} \Xi_{c c}^{++}$ & $-\frac{1}{\sqrt{3}}$ & $\frac{2}{\sqrt{3}}$ & 1 & 0 \\
\hline $\bar{D}^{0} \Omega_{c c}$ & $\frac{1}{\sqrt{3}}$ & $-\frac{2}{\sqrt{3}}$ & 1 & 0 \\
\hline$\eta_{c} \Xi_{c}^{+}$ & $\frac{2 \sqrt{2}}{3}$ & $-\frac{\sqrt{2}}{3}$ & 0 & 0 \\
\hline$\eta_{c} \Xi_{c}^{\prime+}$ & 0 & 0 & 0 & $-\sqrt{2}$ \\
\hline
\end{tabular}


TABLE VIII. The $\alpha$ and $\beta$ coefficients for the meson-baryon (MB) channels involved in the radiative decay of resonances into $\Xi_{c}^{0}$ and $\Xi_{c}^{\prime 0}$. We only show the coefficients for channels with charged particles.

\begin{tabular}{|c|c|c|c|c|}
\hline \multirow[b]{2}{*}{ MB } & \multicolumn{2}{|c|}{$\Xi_{c}^{0}$} & \multicolumn{2}{|c|}{$\Xi_{c}^{\prime 0}$} \\
\hline & $\alpha$ & $\beta$ & $\alpha$ & $\beta$ \\
\hline$\pi^{-} \Xi_{c}^{+}$ & $-\frac{1}{3 \sqrt{2}}$ & $\frac{5}{3 \sqrt{2}}$ & $\frac{1}{\sqrt{6}}$ & $\frac{1}{\sqrt{6}}$ \\
\hline$\pi^{-} \Xi_{c}^{\prime+}$ & $-\frac{1}{\sqrt{6}}$ & $-\frac{1}{\sqrt{6}}$ & $\frac{1}{\sqrt{2}}$ & $-\frac{1}{\sqrt{2}}$ \\
\hline$K^{-} \Lambda_{c}$ & $-\frac{1}{3 \sqrt{2}}$ & $\frac{5}{3 \sqrt{2}}$ & $-\frac{1}{\sqrt{6}}$ & $-\frac{1}{\sqrt{6}}$ \\
\hline$K^{-} \Sigma_{c}^{+}$ & $\frac{1}{\sqrt{6}}$ & $\frac{1}{\sqrt{6}}$ & $\frac{1}{\sqrt{2}}$ & $-\frac{1}{\sqrt{2}}$ \\
\hline$D^{+} \Sigma^{-}$ & $-\frac{2}{\sqrt{3}}$ & $\frac{1}{\sqrt{3}}$ & 0 & -1 \\
\hline$D_{s}^{+} \Xi^{-}$ & $\frac{2}{\sqrt{3}}$ & $-\frac{1}{\sqrt{3}}$ & 0 & -1 \\
\hline$D_{s}^{-} \Xi_{c c}^{+}$ & $-\frac{1}{\sqrt{3}}$ & $\frac{2}{\sqrt{3}}$ & -1 & 0 \\
\hline$D^{-} \Omega_{c c}$ & $\frac{1}{\sqrt{3}}$ & $-\frac{2}{\sqrt{3}}$ & -1 & 0 \\
\hline
\end{tabular}

TABLE IX. The $\alpha$ and $\beta$ coefficients for the meson-baryon (MB) channels involved in the radiative decay of resonances into $\Sigma_{c}^{0}$. We only show the coefficients for channels with charged particles.

\begin{tabular}{lccc}
\hline \hline $\mathrm{MB}$ & $\alpha$ & $\Sigma_{c}^{0}$ & \\
\hline$\pi^{-} \Lambda_{c}$ & $-\frac{1}{\sqrt{3}}$ & & $\beta$ \\
$\pi^{-} \Sigma_{c}^{+}$ & 1 & $-\frac{1}{\sqrt{3}}$ \\
$D_{s}^{+} \Sigma^{-}$ & 0 & -1 \\
$D^{-} \Xi_{c c}^{+}$ & $\sqrt{2}$ & $-\sqrt{2}$ \\
\hline \hline
\end{tabular}

[1] B. Aubert et al. (BABAR Collaboration), Phys. Rev. Lett. 90, 242001 (2003).

[2] R. A. Briere et al. (CLEO Collaboration), Phys. Rev. D 74, 031106 (2006).

[3] P. Krokovny et al. (Belle Collaboration), Phys. Rev. Lett. 91, 262002 (2003).

[4] K. Abe et al. (Belle Collaboration), Phys. Rev. Lett. 92, 012002 (2004).

[5] S. K. Choi et al. (Belle Collaboration), Phys. Rev. Lett. 91, 262001 (2003).

[6] D. E. Acosta et al. (CDF II Collaboration), Phys. Rev. Lett. 93, 072001 (2004).

[7] V. M. Abazov et al. (D0 Collaboration), Phys. Rev. Lett. 93, 162002 (2004).

[8] B. Aubert et al. (BABAR Collaboration), Phys. Rev. D 71, 071103 (2005).

[9] K. Abe et al. (Belle Collaboration), Phys. Rev. Lett. 98, 082001 (2007).

[10] P. Pakhlov et al. (Belle Collaboration), Phys. Rev. Lett. 100, 202001 (2008).

[11] K. Abe et al. (Belle Collaboration), Phys. Rev. Lett. 94, 182002 (2005).

[12] B. Aubert et al. (BABAR Collaboration), Phys. Rev. Lett. 101, 082001 (2008).
TABLE X. The $\alpha$ and $\beta$ coefficients for the meson-baryon (MB) channels involved in the radiative decay of resonances into $\Sigma_{c}^{++}$. We only show the coefficients for channels with charged particles.

\begin{tabular}{lccc}
\hline \hline & & $\Sigma_{c}^{++}$ & \\
$\mathrm{MB}$ & $\alpha$ & $\beta$ \\
\hline$\pi^{+} \Lambda_{c}$ & $-\frac{1}{\sqrt{3}}$ & $-\frac{1}{\sqrt{3}}$ \\
$\pi^{+} \Sigma_{c}^{+}$ & -1 & 1 \\
$\pi^{0} \Sigma_{c}^{++}$ & 1 & -1 \\
$D^{+} p$ & 0 & $-\sqrt{2}$ \\
$K^{+} \Xi_{c}^{+}$ & $\frac{1}{\sqrt{3}}$ & $\frac{1}{\sqrt{3}}$ \\
$\eta \Sigma_{c}^{++}$ & $\sqrt{\frac{2}{3}}$ & $-\sqrt{\frac{2}{3}}$ \\
$K^{+} \Xi_{c}^{\prime+}$ & -1 & 1 \\
$D_{s}^{+} \Sigma^{+}$ & 0 & $-\sqrt{2}$ \\
$\eta^{\prime} \Sigma_{c}^{++}$ & $\frac{1}{\sqrt{3}}$ & $-\frac{1}{\sqrt{3}}$ \\
$\bar{D}^{0} \Xi_{c c}^{++}$ & $-\sqrt{2}$ & 0 \\
$\eta_{c} \Sigma_{c}^{++}$ & 0 & $-\sqrt{2}$ \\
\hline \hline
\end{tabular}

[13] S. Uehara et al. (Belle Collaboration), Phys. Rev. Lett. 96, 082003 (2006).

[14] T. Barnes, F. E. Close, and H. J. Lipkin, Phys. Rev. D 68, 054006 (2003).

[15] E. E. Kolomeitsev and M. F. M. Lutz, Phys. Lett. B 582, 39 (2004).

[16] J. Hofmann and M. F. M. Lutz, Nucl. Phys. A733, 142 (2004).

[17] F. K. Guo, P. N. Shen, H. C. Chiang, and R. G. Ping, Phys. Lett. B 641, 278 (2006).

[18] F. K. Guo, P. N. Shen, and H. C. Chiang, Phys. Lett. B 647, 133 (2007).

[19] Y. J. Zhang, H. C. Chiang, P. N. Shen, and B. S. Zou, Phys. Rev. D 74, 014013 (2006).

[20] F.E. Close and P.R. Page, Phys. Lett. B 578, 119 (2004).

[21] C. Y. Wong, Phys. Rev. C 69, 055202 (2004).

[22] E. S. Swanson, Phys. Lett. B 588, 189 (2004).

[23] D. Gamermann, E. Oset, D. Strottman, and M. J. Vicente Vacas, Phys. Rev. D 76, 074016 (2007).

[24] D. Gamermann and E. Oset, Eur. Phys. J. A 33, 119 (2007).

[25] D. Gamermann and E. Oset, Phys. Rev. D 80, 014003 (2009). 
[26] Y. Q. Chen and X. Q. Li, Phys. Rev. Lett. 93, 232001 (2004).

[27] L. Maiani, F. Piccinini, A. D. Polosa, and V. Riquer, Phys. Rev. D 71, 014028 (2005).

[28] M. E. Bracco, A. Lozea, R. D. Matheus, F. S. Navarra, and M. Nielsen, Phys. Lett. B 624, 217 (2005).

[29] P. Bicudo, Phys. Rev. D 74, 036008 (2006).

[30] B. A. Li, Phys. Lett. B 605, 306 (2005).

[31] M. Artuso et al. (CLEO Collaboration), Phys. Rev. Lett. 86, 4479 (2001).

[32] R. Mizuk et al. (Belle Collaboration), Phys. Rev. Lett. 94, 122002 (2005).

[33] C. P. Jessop et al. (CLEO Collaboration), Phys. Rev. Lett. 82, 492 (1999).

[34] S. E. Csorna et al. (CLEO Collaboration), Phys. Rev. Lett. 86, 4243 (2001).

[35] R. Chistov et al. (Belle Collaboration), Phys. Rev. Lett. 97, 162001 (2006).

[36] B. Aubert et al. (BABAR Collaboration), Phys. Rev. D 77, 012002 (2008).

[37] B. Aubert et al. (BABAR Collaboration), Phys. Rev. Lett. 97, 232001 (2006).

[38] B. Aubert et al. (BABAR Collaboration), Phys. Rev. Lett. 98, 012001 (2007).

[39] R. Mizuk et al. (Belle Collaboration), Phys. Rev. Lett. 98, 262001 (2007).

[40] L. Tolos, J. Schaffner-Bielich, and A. Mishra, Phys. Rev. C 70, 025203 (2004).

[41] M.F. M. Lutz and E. E. Kolomeitsev, Nucl. Phys. A730, 110 (2004).

[42] M. F. M. Lutz and E. E. Kolomeitsev, Nucl. Phys. A755, 29 (2005).

[43] J. Hofmann and M.F. M. Lutz, Nucl. Phys. A763, 90 (2005).

[44] J. Hofmann and M.F. M. Lutz, Nucl. Phys. A776, 17 (2006).

[45] T. Mizutani and A. Ramos, Phys. Rev. C 74, 065201 (2006).

[46] C. Garcia-Recio, V. K. Magas, T. Mizutani, J. Nieves, A. Ramos, L. L. Salcedo, and L. Tolos, Phys. Rev. D 79, 054004 (2009).

[47] C. E. Jimenez-Tejero, A. Ramos, and I. Vidana, Phys. Rev. C 80, 055206 (2009).

[48] D. Gamermann, C. Garcia-Recio, J. Nieves, L. L. Salcedo, and L. Tolos, Phys. Rev. D 81, 094016 (2010).

[49] H. Y. Cheng, C. Y. Cheung, G. L. Lin, Y. C. Lin, T. M. Yan, and H. L. Yu, Phys. Rev. D 47, 1030 (1993).

[50] H. Y. Cheng, Phys. Lett. B 399, 281 (1997).

[51] M. J. Savage, Phys. Lett. B 345, 61 (1995).
[52] M. C. Banuls, A. Pich, and I. Scimemi, Phys. Rev. D 61, 094009 (2000).

[53] S. L.F. Zhu and Y.B.F. Dai, Phys. Rev. D 59, 114015 (1999).

[54] S. Tawfiq, J. G. Korner, and P. J. O’Donnell, Phys. Rev. D 63, 034005 (2001).

[55] M. A. Ivanov, J. G. Korner, V.E. Lyubovitskij, and A. G. Rusetsky, Phys. Rev. D 60, 094002 (1999).

[56] M. A. Ivanov, J. G. Korner, and V. E. Lyubovitskij, Phys. Lett. B 448, 143 (1999).

[57] J. Dey, V. Shevchenko, P. Volkovitsky, and M. Dey, Phys. Lett. B 337, 185 (1994).

[58] Fayyazuddin and Riazuddin, Mod. Phys. Lett. A 12, 1791 (1997).

[59] A. Majethiya, B. Patel, and P. C. Vinodkumar, Eur. Phys. J. A 42, 213 (2009).

[60] P. L. Cho, Phys. Rev. D 50, 3295 (1994).

[61] S. L. Zhu, Phys. Rev. D 61, 114019 (2000).

[62] C. K. Chow, Phys. Rev. D 54, 3374 (1996).

[63] Y. Dong, A. Faessler, T. Gutsche, S. Kumano, and V.E. Lyubovitskij, Phys. Rev. D 82, 034035 (2010).

[64] D. Gamermann, L. R. Dai, and E. Oset, Phys. Rev. C 76, 055205 (2007).

[65] A. Faessler, T. Gutsche, V.E. Lyubovitskij, and Y. L. Ma, Phys. Rev. D 76, 014005 (2007).

[66] M.F. M. Lutz and M. Soyeur, Nucl. Phys. A813, 14 (2008).

[67] Y. Dong, A. Faessler, T. Gutsche, and V. E. Lyubovitskij, J. Phys. G 38, 015001 (2011).

[68] W. H. Liang, R. Molina, and E. Oset, Eur. Phys. J. A 44, 479 (2010).

[69] R. Molina, H. Nagahiro, A. Hosaka, and E. Oset, arXiv:1009.4881.

[70] T. Branz, R. Molina, and E. Oset, arXiv:1010.0587.

[71] C. Amsler et al. (Particle Data Group), Phys. Lett. B 667, 1 (2008).

[72] R. Machleidt, K. Holinde, and C. Elster, Phys. Rep. 149, 1 (1987).

[73] R. K. Logan and H.W. Wyld, Phys. Rev. 158, 1467 (1967).

[74] B. Borasoy, P. C. Bruns, U. G. Meissner, and R. Nissler, Phys. Rev. C 72, 065201 (2005).

[75] M. Doring, E. Oset, and S. Sarkar, Phys. Rev. C 74, 065204 (2006).

[76] M. Doring, Nucl. Phys. A786, 164 (2007).

[77] L. S. Geng, E. Oset, and M. Doring, Eur. Phys. J. A 32, 201 (2007).

[78] E. Oset and A. Ramos, Nucl. Phys. A635, 99 (1998). 